\title{
REVIEW \\ Maintenance of an undifferentiated state of human induced \\ pluripotent stem cells through migration-dependent regulation of the balance between cell-cell and cell-substrate interactions
}

\author{
Mee-Hae Kim and Masahiro Kino-oka* \\ Department of Biotechnology, Graduate School of Engineering, Osaka University, 2-1 Yamadaoka, \\ Suita, Osaka 565-0871, Japan \\ * Corresponding author. \\ Tel.: +81-(0)6-6879-7444; fax: +81-(0)6-6879-4246 \\ E-mail address: kino-oka@bio.eng.osaka-u.ac.jp (M. Kino-oka). \\ Running title: Maintenance of undifferentiated hiPSCs
}




\section{ABSTRACT}

We present an outlook on the current strategies for maintaining and culturing human induced pluripotent stem cells (hiPSCs) in an undifferentiated state without affecting their pluripotency. First, cell structures and function are described in relation to interactions between hiPSCs and their surroundings. Second, the phenomenon of spontaneous deviation from undifferentiated hiPSCs in feeder cell-dependent cultures is addressed, with a summary of current topics that are of particular interest to our studies. The key regulatory factors that can contribute to the growth and maintenance of undifferentiated hiPSCs are also discussed, with a summary of recent work toward a culture strategy to control hiPSC fate through balancing cell-cell and cell-substrate interactions. Finally, we discuss culture process design in our previous studies with respect to maintaining and expanding cultures of undifferentiated and pluripotent hiPSCs. We focus on the regulation of migration-dependent balance between cell-cell and cell-substrate interactions. This review offers novel insights into the regulation and processing of stem cells for research in regenerative medicine.

Keywords: Human induced pluripotent stem cells; Undifferentiated state; Cell migration; Cell-cell interaction; Cell--substrate interaction 


\section{INTRODUCTION}

Induced pluripotent stem cells (iPSCs) are generated from various somatic cells by exogenous expression of defined transcription factors (Oct4, Sox2, Klf4, and c-Myc) that confer properties similar to those of embryonic stem cells (ESCs) $(1,2)$. Since iPSCs resemble ESCs in their ability to generate cells of all three germ layers, patient-specific iPSCs could resolve the ethical and histo-incompatibility issues surrounding the use of ESCs. iPSCs exhibit ESC-like properties in terms of morphology, proliferation, differentiation capacity, and genomic and epigenomic states (3, 4), suggesting that iPSCs could be regarded as an infinite cell source for many areas of research and medicine.

The expansion of iPSCs remains a challenging process because spontaneous differentiation is accompanied by loss of self-renewal and pluripotent qualities $(5,6)$. Therefore, iPSCs require a specialized growth environment to retain an undifferentiated phenotype. The components of this optimal growth environment are constantly undergoing revisions and improvements. To facilitate development of in vitro culture systems, many researchers have attempted to design microenvironments that mimic the stem cell niche, thereby driving cells down their preferred self-renewal and differentiation pathway (5-10). A typical strategy is to enrich the biochemical environment of the in vitro culture media with a combination of soluble growth factors, cytokines, and/or serum protein to achieve successful maintenance of the undifferentiated cell state after several passages $(11,12)$. In addition to enhancing the soluble biochemical signal, gaining a fundamental understanding of the cell-cell and cell-substrate interactions is required to understand, design, and refine these artificial environments $(13,14)$. A comprehensive understanding of these interactions will allow for the effective design and development of materials capable of influencing iPSC self-renewal and the lineage into which these cells differentiate. This requires a multidisciplinary approach, integrating various concepts in the stem cell culture process.

In this review, we have focused on recent advances in culture strategies aimed at expanding populations of undifferentiated human iPSCs (hiPSCs), together with approaches to studying the 
1 mechanisms underlying their self-renewal and pluripotency. We discuss morphological and

2 biological features of cellular structures and mechanisms related to cell-cell and cell-substrate

3 interactions. We then introduce principles for creating cell culture bioprocesses by considering the

4 mechanisms of cell-cell and cell-substrate interactions, and delineate the prospects of surface-based

5 culture strategies for clinical application of hiPSCs in the future.

\section{UNDERSTANDING THE CELLULAR STRUCTURES RELATED TO CELL-CELLAND}

\section{CELL-SUBSTRATE INTERACTIONS}

An understanding of the mechanisms that control self-renewal, apoptosis, and commitment to differentiation of hiPSCs is important for developing improved methods of culture and differentiation that could be successfully used in clinical practice. There are three different cell fates that occur within hiPSC colonies during maintenance of cultures (Fig.1). Typically, hiPSC colonies Fig. 1 grow as a monolayer and sustain an undifferentiated state. Undifferentiated hiPSCs form compact colonies of tightly associated with cells via cadherin-mediated cell-cell interactions $(12,15)$, exhibiting a small, cobblestone-like morphology. With longer culture, the spontaneous occurrence associated with apoptotic cell death at the central region accelerated the morphological transformation of undifferentiated cells to deviated cells as the culture became prolonged, leading to formation of deviated cells from the undifferentiation at the center of the colony. Some colonies display a double-ring pattern, with large and flattened cells appearing at the center or periphery of the colonies. Once deviation occurs in these colonies, apoptosis triggers rapid invasion of the space between colonies in long-term hiPSC cultures.

Cadherin- and integrin-mediated interactions are known to influence differentiation during maintenance of ESCs and iPSCs $(8,15,16)$. E-cadherin has been identified as a key factor in the regulation of pluripotency and self-renewal signaling pathways in human ESCs (hESCs) and hiPSCs. The cytoplasmic domain of E-cadherin contains binding regions for $\beta$-catenin, p120-catenin, and various other regulatory elements including phosphorylation sites and regions 
1 recognized by the degradation machinery of the cell (17-19). Interaction of E-cadherin with

$2 \beta$-catenin and p120-catenin facilitates stabilization of the cytoplasmic cell adhesion complex,

3 protecting it from degradation and maintaining anchorage of E-cadherin to the actin cytoskeleton.

4 E-cadherin expression further maintains the self-renewal capacity of hESCs by influencing the

5 expression of Oct3/4 and Nanog genes (6). Altered levels of E-cadherin expression in monolayer

6 cultures occurs spontaneously, along with apoptosis and differentiation. Down-regulation of

7 E-cadherin stimulates pro-apoptotic Caspase-3 and suppresses the apoptotic inhibitory gene $B c l-X L$,

8 resulting in increased level of cell death $(12,20,21)$. Li et al. (15) observed that $>98 \%$ of individual

9 hESCs that failed to maintain E-cadherin expression following single cell dissociation exhibited cell

10 death or differentiation within $72 \mathrm{~h}$ of seeding. Subsequent up-regulation of E-cadherin expression

11 tended to result in aggregated colonies with an embryoid body-like structure, suggesting that

12 adhesion between cells is preferable to that between cells and the culture substratum. Close contact

13 between cells in these aggregates could lead to paracrine interactions that could generate spatial

14 heterogeneity, and trigger differentiation.

15 Integrin-mediated interactions are also considered essential for maintenance of stem cell

16 survival, pluripotency, and growth. The maintenance of ESCs and iPSCs requires extracellular

17 matrix proteins such as laminin and fibronectin (22). Fate decisions of hiPSCs are reportedly

18 supported by various integrin-mediated signal pathways. Among them, the PI3K-Akt pathway has

19 been shown to inhibit apoptosis and attenuate cell death after hESC dissociation, with constitutive

20 overexpression of Akt partially improving hESC survival $(12,22)$. Li et al. (16) also demonstrated

21 that functional interaction between the small Rho family GTPase Rac1 and E-cadherin is

22 responsible for regulating hESC self-renewal (20). This ability of the PI3K-Akt pathway to promote

23 cell survival indicates a broader role for Rac1-related GTPases in the regulation of cell survival or

24 death decisions. E-cadherin-mediated interactions are commonly associated with $\beta$-catenin

25 signaling, and can also stimulate Rac-PI3K-Akt signaling in hESCs (8, 23). Therefore,

26 integrin-mediated signaling pathways are of interest in the maintenance of hiPSC pluripotency. 
The mechanism underlying how crosstalk between cadherin- and integrin-mediated

2 interactions affect cell fate decisions such as cell survival, self-renewal and pluripotency, in hESCs

3 and hiPSCs during maintenance culture have only begun to be understood in recent years (12).

4 Numerous studies have revealed that multiple regulatory mechanisms are involved in the interplay

5 between E-cadherin and integrin in the regulation of $\mathrm{hESC}$ fate $(8,15,16,24)$. Several key

6 mechanistic elements participate in a range of regulatory events in hESCs, including cooperation of

7 the actin cytoskeleton with Rho family GTPases, regulation of E-cadherin expression at the hESC

8 surface by turnover and membrane trafficking, and interplay between E-cadherin and integrin (25,

9 26). The inhibition of endogenous Racl activity by a specific inhibitor also increased the quantity of

10 E-cadherin/ $\beta$-catenin complexes. These findings point to the ability of endogenous Rac1 to

11 modulate E-cadherin and its function. Although the exact mechanisms by which these factors permit

12 the propagation and maintenance of undifferentiated hiPSCs remain unclear, mounting evidence

13 suggests that they play a crucial role in the growth and maintenance of undifferentiated hiPSCs.

\section{WHY DO HIPSCS DEVIATE FROM AN UNDIFFERENTIATED STATE?}

We highlight how a stem cell "decides" whether to survive, self-renew or differentiate, and discuss how this information can be used to develop novel strategies for controlling their fate. The distinct qualities that allow assessment of the phenomenon of deviation from undifferentiated hiPSCs in an in vitro culture environment deserve particular attention. Undifferentiated hiPSCs are expanded as monolayer colonies in adhesion culture in the presence of feeder cells. Culturing with mouse or human feeder cells is the most widely used method of providing the soluble factors necessary to support the growth and maintenance of undifferentiated hiPSCs $(1,2,27)$. We have previously proposed possible mechanisms that control hiPSC self-renewal under in vitro culture conditions using SNL and MEF feeder cells (28). For both types of feeder cells, the most important factors influencing the deviation of hiPSC colonies from an undifferentiated state involved cell migration and growth inside a single colony. The process of deviation from undifferentiated hiPSCs 
1 during culture has been outlined (Fig. 2). Colonies of hiPSCs cultured with SNL feeder cells grew

2 outward, dividing while migrating on the surface, and gradually became larger and more tightly

3 packed during culture (Fig. 2A). Motility decreased steadily with increasing population density at

4 the center of colony, and the cells overlapped, indicating that cells at the central region of the

5 colony partially detach from the substrate. These cells in the central region of large growing

6 colonies exhibited morphological changes accompanying apoptosis, and proliferated while

7 undergoing transition from small cells to large and flattened cells. As a result, a large number of hiPSCs within colonies dissociated from one another and spread out as single cells. It appears that the contraction of alone blebbing apoptotic cell could act to drag neighbor cells into the space vacated by the dying cell, thereby spawning deviated cells in the central region. These observations 11 provided important information toward understanding the process of deviation from an undifferentiated state, which occurs at the center of hiPSC colonies. They suggest that a delicate interaction between adjacent cells and their substrate, along with colony growth, is closely related to the occurrence, replication, and transformation of deviated cells during cell expansion. This could explain why the peripheries of undifferentiated colonies displayed enhanced migratory activity, as there would be an extended time-lag before newly-divided daughter cells are ready to divide again.

17 Conversely, deviation from the undifferentiated state in colonies cultured with MEF feeder 18 cells involved a rim of rapidly proliferating and outwardly migrating cells at the periphery of the 19 colony. These cells underwent rapid division in subsequent cultures (Fig. 2B). It is most likely that 20 the enhanced migration of hiPSCs in response to MEF feeder cells reduce cell-cell interactions at 21 the peripheral region of colonies. It might eventually be possible to facilitate expansion of deviated cells in growing hiPSC colonies. These cells were characterized by a loss of E-cadherin-mediated cell-cell interactions and acquisition of a more motile phenotype. These observations suggest that cells at the periphery of the colonies cultured with MEF feeder cells disrupt cell-cell interactions through accidental events during migration. This migration led to accelerated replication of deviated 
undifferentiated hiPSCs. Migration should be considered as a key parameter in the determination of hiPSC fate.

Based on these findings, we propose that the observed morphological changes occur because altered migration changes the balance between cell-cell and cell-substrate interactions. Ultimately an appropriate balance between interactions of cell-cell and cell-substrate leads to the formation of undifferentiated colonies. When cell-cell interactions overcome cell-substrate interactions, colonies with deviated cells form in the central region (Fig. 2A). Conversely, when cell-substrate interactions overcome cell-cell interactions, colonies form with deviated cells at the periphery (Fig. 2B). A major reason for the differences in migratory capacities could be the response of hiPSCs to two types of feeder cells in culture environments. These differences might be crucial in determining the point of occurrence for deviated cells from the undifferentiated state. Additionally, we investigated how the underlying pattern of colony formation affects the balance between cell-cell and cell--substrate interactions by inhibition of migration in cultures of feeder cells. In culture with MEF feeder cells, blocking Rac1 activation with a specific Rac1 inhibitor NSC23766, revealed that the deviation of undifferentiated hiPSCs resulted in predominantly deviated cells in the central region than at the peripheral region. Deviation of hiPSC colonies that were not treated with NSC23766, remained concentrated at the periphery of colonies (Fig. 2C). These studies suggest that cell-cell and cell-substrate interactions influence migration during maintenance of undifferentiated hiPSCs, and provide a resource for future studies of hiPSCs.

\section{NEW INSIGHTS INTO THE ROLES OF MIGRATION IN MAINTENANCE OF}

\section{UNDIFFERENTIATED HIPSCS}

The interaction of stem cell with specific microenvironmental elements is thought to be a key regulatory mechanism in the maintenance of self-renewal and differentiation capacity $(7,8)$. The regulation of stem cell fate has been shown to be influenced by cues provided by reciprocal interactions with an artificial extracellular microenvironment, comprising soluble bioactive agents 
1 and the synthetic substrates (14). Another aspect of the microenvironment that has been mimicked

2 in the study of stem cell fate is the regulation of behaviors such as morphology and migration.

3 However, there are few reports focusing on culture strategies for developing and maintaining hiPSC

4 cultures that are based on their dynamic behavior.

5 In our recent studies, we addressed possible mechanisms for controlling hiPSC fate decisions

6 by influencing the balance between cell-cell and cell-substrate interactions through altered cell

7 migration (29). We have also discussed recent insights concerning the maintenance of

8 undifferentiated hiPSCs through the regulation of migration (Fig. 3). Deviation from an Fig. 3

9 undifferentiated state by hiPSCs in colonies cultured with SNL feeder cells involve decreased

10 migration during colony formation, and induction of apoptosis at the center of colony. The

11 deviation of undifferentiated hiPSCs cultured with SNL feeder cells occurred spontaneously in cells

12 at the center of the colony, and was dependent on colony size. We have investigated dendrimer

13 surfaces with dendron structure and their regulation of cell morphology by changing the generation

14 number of dendrimers, and attempted to clarity the relationship between cell morphology and

15 function on these designed surfaces. The cell morphological changes on dendrimer surface were

16 supposed, including the ligand display control over cells which is caused by the binding balance

17 between integrin on the cytoplasm and adhesion protein on surface, to topographical control over

18 cells which is nano-topography of dendron structure, and to surface stiffness control over cells (29,

19 30). Colony morphology was either scattered or aggregated, depending on the levels of migration.

20 An appropriate migration pattern leads to formation of an undifferentiated hiPSC colony. When

21 migration level increased, colony morphology appeared to be scattered and was associated with

22 apoptosis. Further migration induces aggregate formation, resulting in differentiation toward cells

23 of the early mesoderm. Modulating survival versus death, and pluripotency versus differentiation,

24 during cell fate decisions-making can therefore be explained in terms of the balance between cell-

25 cell and cell-substrate interactions by altering cell migration.

26 In a recent study, we examined the morphological and functional responses of hiPSCs to a 
1 dendrimer surface (30). We investigated the expression of genes associated with the undifferentiated

2 state of hiPSCs (Oct3/4, Sox2, and Nanog), and the genes associated with lineage commitment in

3 hiPSCs cultured on the first-generation dendrimer surface or on a gelatin-coated surface with feeder

4 cells. We successfully maintained two hiPSC lines on dendrimer surfaces over 10 passage (Fig. 4). Fig. 4

5 Semi-quantitative reverse-transcriptase polymerase chain reaction data demonstrated that there were

6 no significant differences between the hiPSCs cultured on the dendrimer surface compared with

7 those cultured on the gelatin-coated surface. Cell commitment to differentiation was evident in the

8 hiPSCs on the dendrimer surface, as genetic markers indicative of the three germ layers were not

9 activated in hiPSCs on either the dendrimer surface or the gelatin-coated surface. Pluripotent

10 signaling networks in hiPSCs are tightly controlled via the coordinated regulation between

11 cadherin- and integrin-mediated interactions. Small perturbations in these pathways are sufficient to

12 regulate maintenance of the undifferentiated state. Application of this culture surface is a useful

13 alternative for obtaining cell preparation enriched with hiPSCs in an undifferentiated state.

14 One important factor to consider is that hiPSC culture occurs in a transient environment with

15 bidirectional signaling between cells and substrate. However, many culture surfaces developed to

16 date might not be conducive to incorporating this dynamic behavior and therefore might not truly

17 mimic the extracellular environment. Cell behavior is constantly governed by instructive

18 biochemical and biophysical cues from the environment. Likewise, cells are continuously

19 remodeling their surrounding environment. The required design of synthetic substrates varies across

20 different cell types, and synthetic environments should be individually generated with distinct cell

21 lineages in mind. Our studies can serve as a culture model to maintain an undifferentiated state of

22 hiPSCs through balance between cell-cell and cell-substrate interactions by altering cell migration,

23 and we believe they also pave the way in the design of suitable culture mediums and substrates

24 while preserving undifferentiated hiPSCs. 

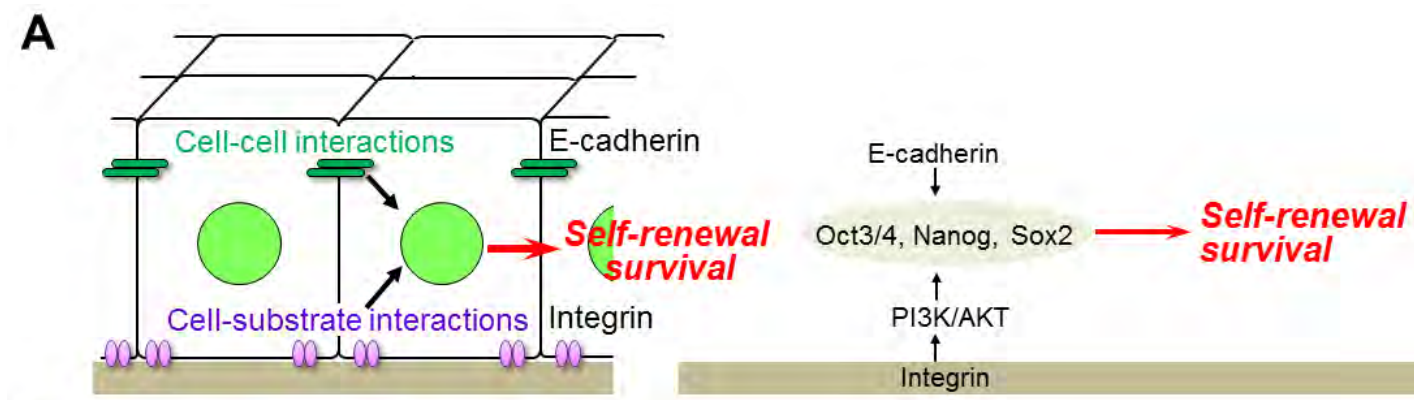

B

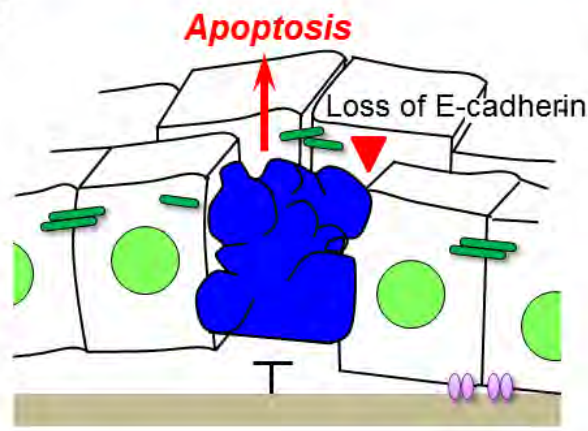

Loss of E-cadherin
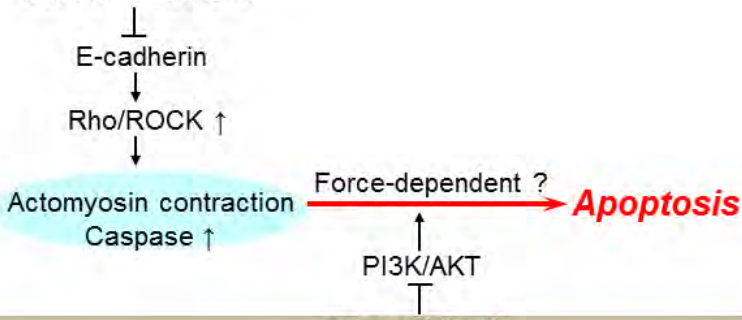

Loss of integrin

C
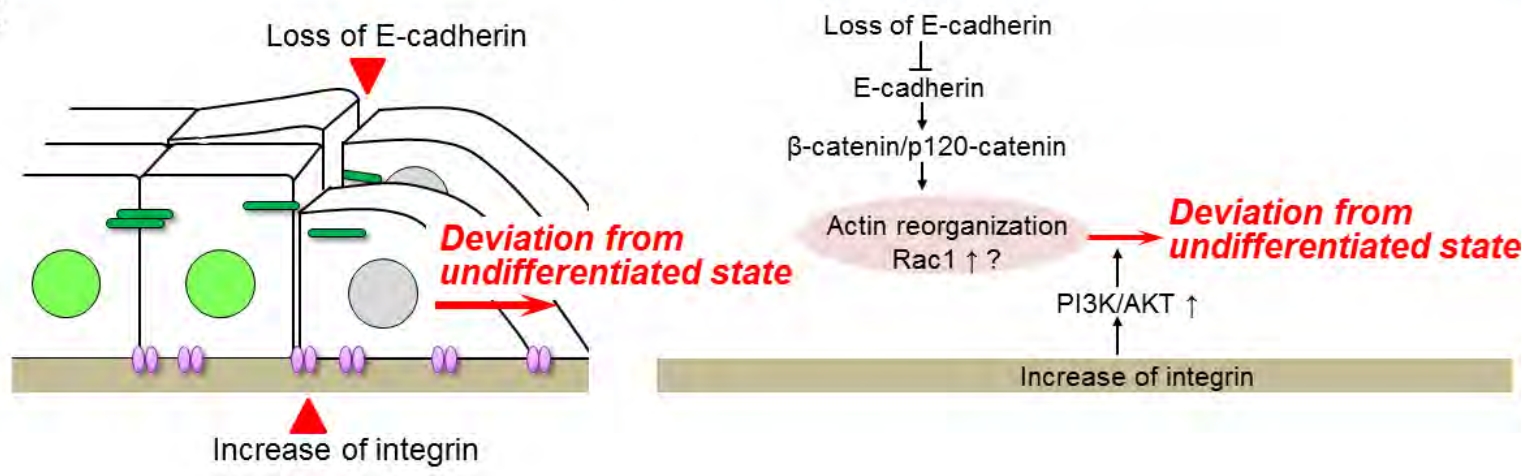

\section{CONCLUSIONS}

We outlined strategies for the expansion of undifferentiated hiPSCs that are well-suited for

4 understanding and regulating the balance between cell-cell and cell-substrate interactions in feeder

5 cell-dependent cultures. We suggest that changes in migrations lead to guidance of hiPSC fate

6 decisions in cultures with two types of feeder cells. Moreover, we found that the coordinated

7 regulation of balance between cell-cell and cell-substrate interactions was important for controlling

8 cell fate decisions such as survival, self-renewal, and pluripotency. Endogenous Rac1 and

9 E-cadherin signaling pathways form an intrinsic key for the regulation of hiPSC fate decisions. Our

10 results from various studies provide insight into the design of culture media and substrates, based 
1 on interactions between the microenvironment and cells. These could serve as useful alternatives for

2 obtaining cell preparations enriched with cells in an undifferentiated or desired differentiation state.

3 Our proposed method offers new strategies of culture process design for the expansion of 4 undifferentiated hiPSCs.

\section{ACKNOWLEDGMENTS}

6 This work was supported in part by the Japan Society for the Promotion of Science (JSPS)

7 KAKENHI, Grants-in-Aid for Young Scientists (B) (Nos. 23760749 and 25820401) and

8 Grant-in-Aid for Scientific Research (C) (No.24592792). Our work was also supported by the

9 "Development of cell manufacturing and processing system for industrialization of regenerative 10 medicine" (No. P14006), which was commissioned by the New Energy and Industrial Technology

11 Development Organization (NEDO). 


\section{References}

1. Takahashi, K., Tanabe, K., Ohnuki, M., Narita, M., Ichisaka, T., Tomoda, K., and Yamanaka, S.: Induction of pluripotent stem cells from adult human fibroblasts by defined factors, Cell, 131, 861-872 (2007).

2. Takahashi, K. and Yamanaka, S.: Induction of pluripotent stem cells from mouse embryonic and adult fibroblast cultures by defined factors, Cell, 126, 663-676 (2006).

3. Pei, D., Xu, J., Zhuang, Q., Tse, H.F., and Esteban, M.A.: Esteban, Induced pluripotent stem cell technology in regenerative medicine and biology, Adv. Biochem. Eng. Biotechnol., 123, 127-141 (2010).

4. Wernig, M., Meissner, A., Foreman, R., Brambrink, T., Ku, M., Hochedlinger, K., Bernstein, B.E., and Jaenisch, R.: In vitro reprogramming of fibroblasts into a pluripotent ES-cell-like state, Nature, 448, 318-324 (2007).

5. Lutolf, M.P., Gilbert, P.M., Blau, H.M.: Designing materials to direct stem-cell fate, Nature 462, 433-441 (2009).

6. Metallo, C.M., Mohr, J.C., Detzel, C.J., de Pablo, J.J., Van Wie, B.J., and Palecek, S.P.: Engineering the stem cell microenvironment, Biotechnol. Prog., 23(1), 18-23 (2007).

7. Fuchs, E., Tumbar, T., and Guasch, G.: Socializing with the neighbors: Stem cells and their niche, Cell, 116(6), 769-778 (2003).

8. Liu, N., Lu, M., Tian, X., and Han, Z.: Molecular mechanisms involved in self-renewal and pluripotency of embryonic stem cells, J. Cell Physiol., 211(2), 279-286 (2007).

9. Yao, S., Chen, S., Clark, J., Hao, E., Beattie, G.M., Hayek, A., and Ding, S.: Long-term self-renewal and directed differentiation of human embryonic stem cells in chemically defined conditions, Proc. Natl. Acad. Sci. USA, 103, 6907-6912 (2006).

10. Watt, F.M. and Hogan, B.L.: Out of Eden: stem cells and their niches. Science, 287, 14271430 (2000).

11. Skottman, H. and Hovatta, O.: Culture conditions for human embryonic stem cells, 
Reproduction, 132(5), 691-698 (2006).

12. Xu, Y., Zhu, X., Hahm, H.S., Wei, W., Hao, E., Hayek, A., and Ding, S.: Revealing a core signaling regulatory mechanism for pluripotent stem cell survival and self-renewal by small molecules, Proc. Natl. Acad. Sci. USA, 107, 8129-8134 (2010).

13. Prowse, A.B., Doran, M.R., Cooper-White, J.J., Chong, F., Munro, T.P., Fitzpatrick, J., Chung, T.L., Haylock, D.N., Gray, P.P., and Wolvetang, E.J.: Long term culture of human embryonic stem cells on recombinant vitronectin in ascorbate free media, Biomaterials, 31, $8281-8288$ (2010).

14. Ross, A.M., Nandivada, H., Ryan, A.L., and Lahann, J.: Synthetic substrates for long-term stem cell culture, Polymer, 53, 2533-2539 (2012).

15. Li, L., Bennett, S.A., and Wang, L.: Role of E-cadherin and other cell adhesion molecules in survival and differentiation of human pluripotent stem cells, Cell Adh. Migr., 6(1):59-70 (2012).

16. Li, L., Wang, S., Jezierski, A., Moalim-Nour, L., Mohib, K., Parks, R.J., Retta, S.F., and Wang, L.: A unique interplay between Rap1 and E-cadherin in the endocytic pathway regulates self-renewal of human embryonic stem cells, Stem Cells, 28, 247-257 (2010).

17. Balzac, F., Avolio, M., Degani, S., Kaverina, I., Torti, M., Silengo, L., Small, J.V., and Retta, S.F.: E-cadherin endocytosis regulates the activity of Rap1: a traffic light GTPase at the crossroads between cadherin and integrin function, J. Cell Sci., 18, 4765-4783 (2005).

18. de Beco, S., Gueudry, C., Amblard, F., and Coscoy, S.: Endocytosis is required for E-cadherin redistribution at mature adherens junctions, Proc. Natl. Acad. Sci. USA, 106, 70107015 (2009).

19. Le, T.L., Yap, A.S., and Stow, J.L.: Recycling of E-cadherin: a potential mechanism for regulating cadherin dynamics, J. Cell Biol., 146, 219-232 (1999).

20. Ohgushi, M. and Sasai, Y.: Lonely death dance of human pluripotent stem cells: ROCKing between metastable cell states, Cell, 21(5), 274-282 (2011). 
1 21. Watanabe, K., Ueno, M., Kamiya, D., Nishiyama, A., Matsumura, M., Wataya, T.,

Takahashi, J.B., Nishikawa, S., Nishikawa, S., Muguruma, K., and Sasai, Y.: A ROCK inhibitor permits survival of dissociated human embryonic stem cells, Nat. Biotechnol., 25, 681-686 (2007).

22. Rowland, T.J., Miller, L.M., Blaschke, A.J., Doss, E.L., Bonham, A.J., Hikita, S.T., Johnson, L.V., and Clegg, D.O.: Roles of integrins in human induced pluripotent stem cell growth on Matrigel and vitronectin, Stem Cells Dev., 19, 1231-1240 (2010).

23. Murga, C., Zohar, M., Teramoto, H., and Gutkind, J.S.: Rac1 and RhoG promote cell survival by the activation of PI3K and Akt, independently of their ability to stimulate JNK and NF-B, Oncogene, 21(2), 207-216 (2002).

24. Noren, N.K., Liu, B.P., Burridge, K., and Kreft, B.: p120 catenin regulates the actin cytoskeleton via Rho family GTPases, J. Cell Biol., 150(3), 567-580 (2000).

25. Kaibuchi K, Kuroda S, Fukata M, and Nakagawa M.: Regulation of cadherin-mediated cell-cell adhesion by the Rho family GTPases, Curr. Opin. Cell Biol., 11(5), 591-596 (1999).

26. Kuroda S, Fukata M, Nakagawa M, and Kaibuchi K.: Cdc42, Rac1, and their effector IQGAP1 as molecular switches for cadherin-mediated cell-cell adhesion, Biochem. Biophys. Res. Commun., 262(1), 1-6 (1999).

27. Keshel, S.H., Soleimani, M., Tavirani, M.R., Ebrahimi, M., Raeisossadati, R., Yasaei, H., Afsharzadeh, D., Behroz, M.J., Atashi, A., Amanpour, S., Khoshzaban, A., Roozafzoon, R., and Behrouzi, G.R.: Evaluation of unrestricted somatic stem cells as a feeder layer to support undifferentiated embryonic stem cells, Mol. Reprod. Dev., 79, 709-718 (2012).

28. Kim, M.-H., Masuda, E., and Kino-oka, M.: Kinetic analysis of deviation from the undifferentiated state in colonies of human induced pluripotent stem cells on feeder layers, Biotechnol. Bioeng., 111, 1128-1138 (2014).

29. Kim, M.-H. and Kino-oka, M.: Switching between self-renewal and lineage commitment of human induced pluripotent stem cells via cell-substrate and cell-cell interactions on a 
1 dendrimer-immobilized surface, Biomaterials, 35, 5670-5678 (2014).

2 30. Kim, M.-H. and Kino-oka, M.: Maintenance of undifferentiated state of human induce

3 d pluripotent stem cells through cytoskeleton-driven force acting to secreted fibronectin

4 on a dendrimer-immobilized surface, J. Biosci. Bioeng., http://dx.doi.org/10.1016/j.jbiosc.2

$5 \quad$ 014.05.011 (available online 16 Jun 2014). 


\section{$1 \quad$ Figure legends}

2 FIG. 1. Schematics depicting the potential involvement of multiple pathways during hiPSC fate determination in feeder cell-dependent cultures. (A) Self-renewal. (B) Apoptosis. (C) Deviation from the undifferentiated state.

FIG. 2. Schematic outlining our hypothesis that two types of hiPSC colonies with deviated cells form in cultures with feeder cells. Colonies with deviated cells in cultures grown on SNL feeder cells (A) and MEF feeder cells (B) formed in the central and peripheral regions through slow and fast migration, respectively. A1-A4 and B1-B4 show the deviation of hiPSC colonies in cultures with SNL and MEF feeder cells, respectively. (C) The Rac1 inhibitor, NSC23766 (100 $\mu \mathrm{M})$, was added to cultures grown on MEF feeder cells. Deviated cells occurred predominantly in central region of colonies through reduced migration.

FIG. 3. Schematic showing regulation of hiPSC fate decision between the undifferentiated state and deviated state with relation to the balance between cell-cell and cell-substrate interactions in cultures grown on feeder cells (Ref. 29; modified).

FIG. 4. Long-term maintenance of undifferentiated hiPSCs (Tic and 201B7 hiPSC lines) cultured on a first-generation dendrimer surface or a gelatin-coated surface with SNL feeder cells. We conducted conventional RT-PCR analysis for undifferentiated stem cell markers and early commitment to differentiation markers after passage 10 . 
A
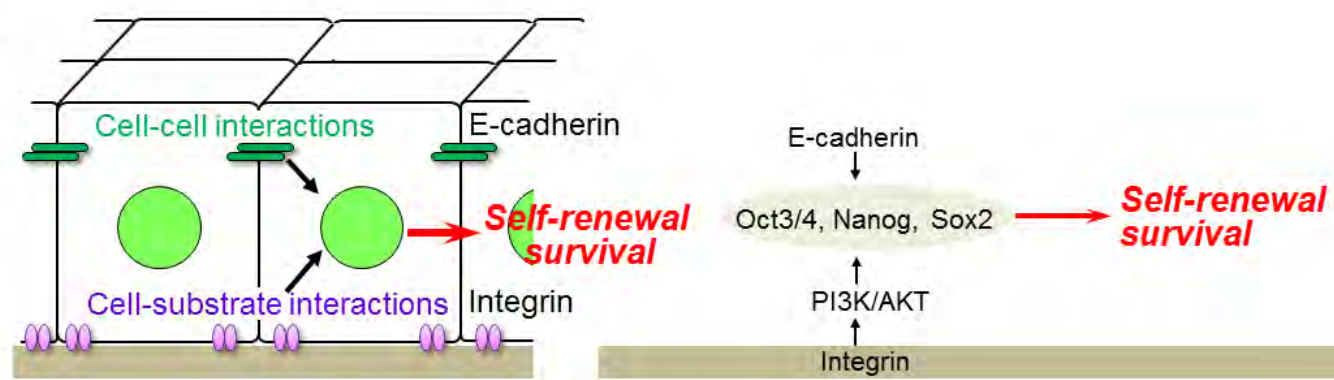

B

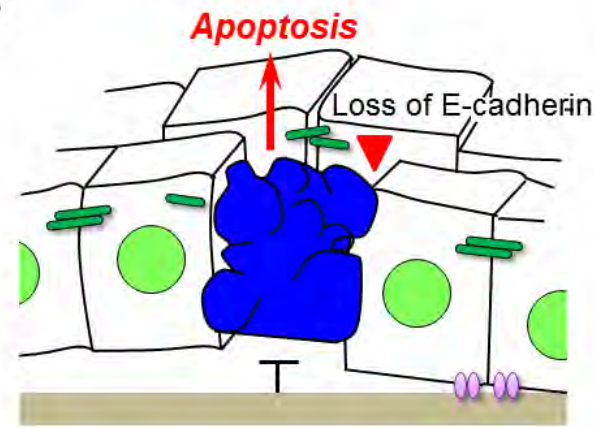

Loss of E-cadherin

$$
\perp
$$

E-cadherin

$\downarrow$

Rho/ROCK $\uparrow$

$\downarrow$

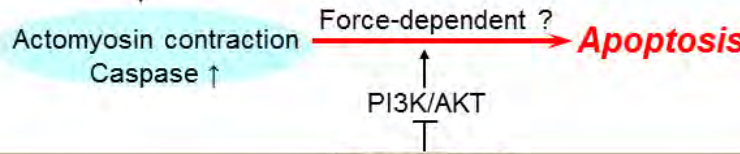

Loss of integrin

C

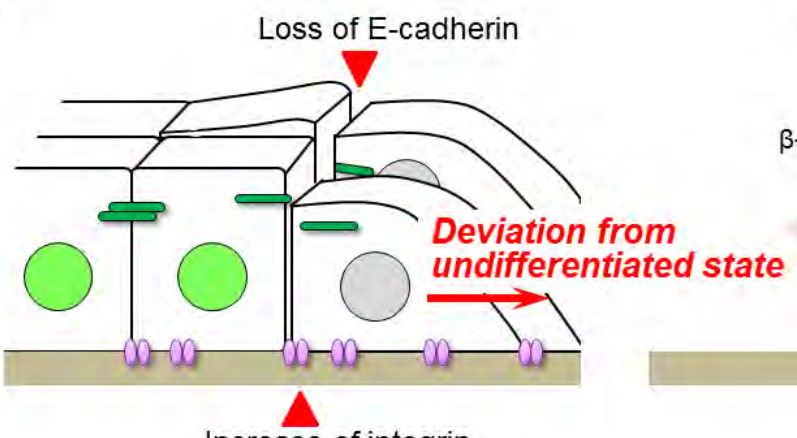

Loss of E-cadherin<smiles>[CH]1C[CH-]C1</smiles>

E-cadherin

$\downarrow$

-catenin/p120-catenin

$\downarrow$

Actin reorganization

Rac1个?

Deviation from

Deviation from PISKIAKT $\uparrow$

Increase of integrin

Figure 1 

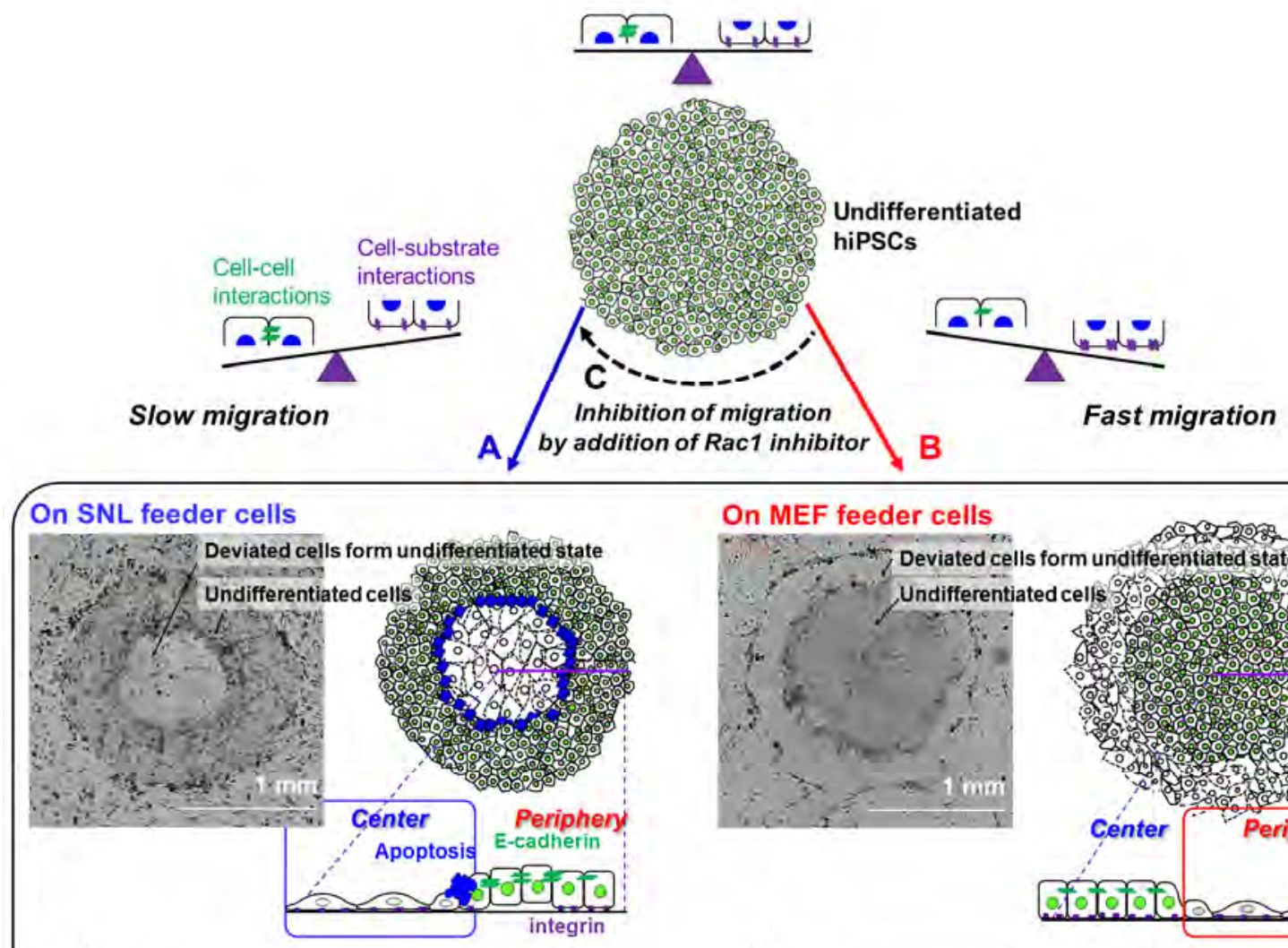

A1

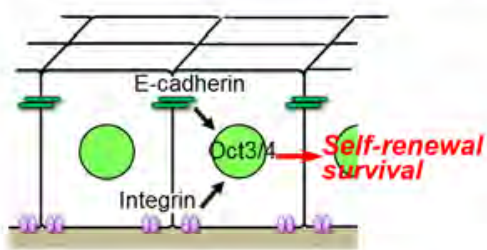

A2

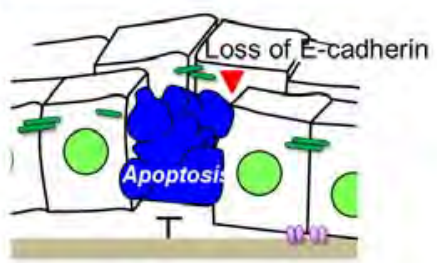

A3

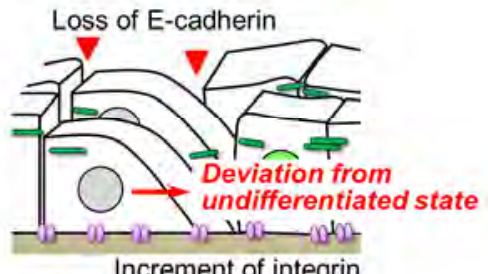

A4

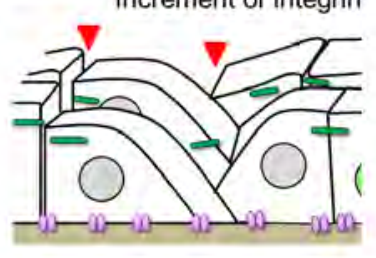

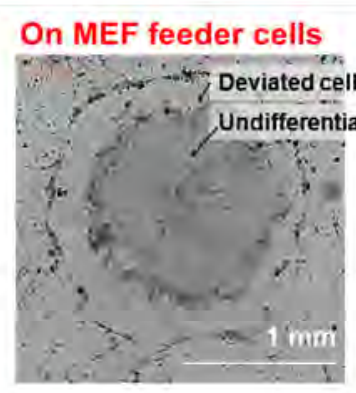

ils form undifferentiated states

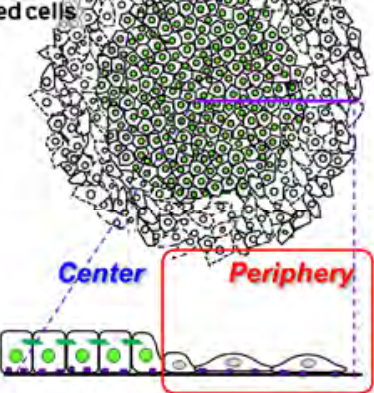

B1

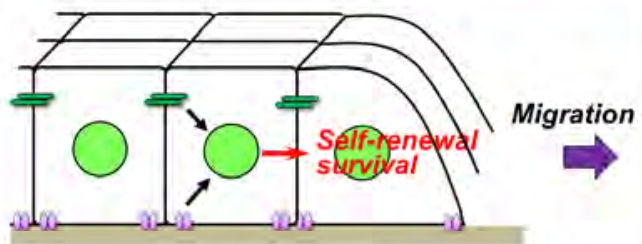

B2

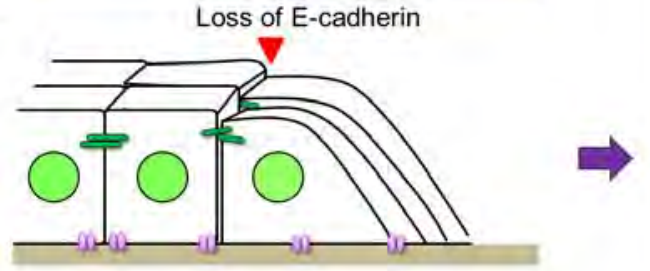

B3

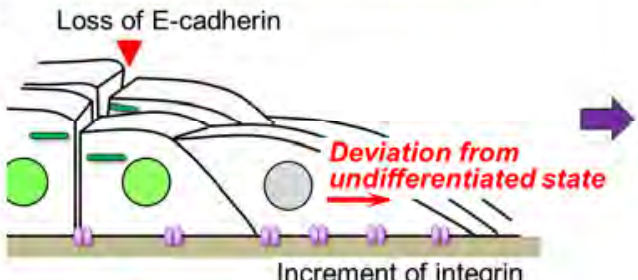

B4

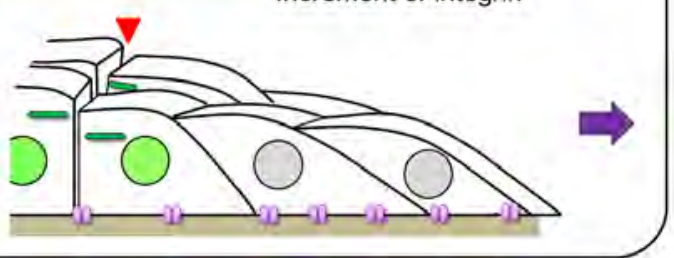

Figure 2 


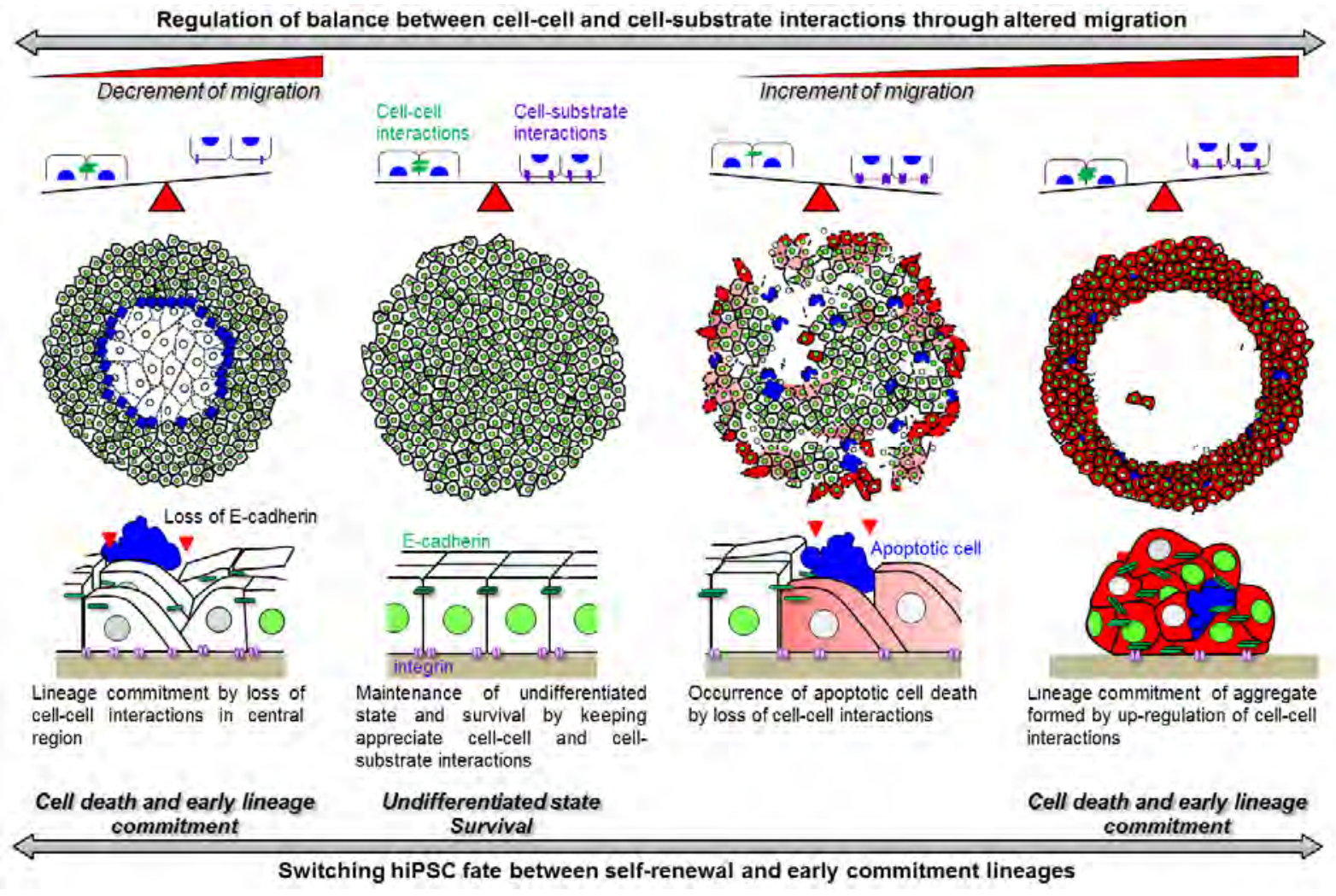

2 Figure 3 


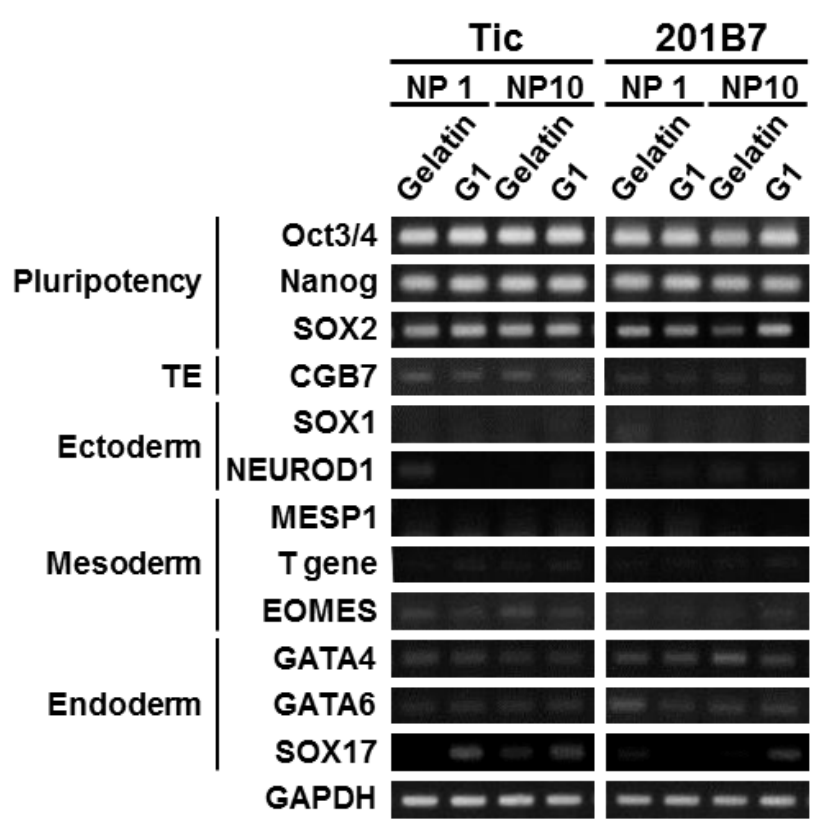

1

$2 \quad$ Figure 4 